\title{
Protocole de mesure directe de l'uranium dans les urines par ICP-MS
}

\author{
J. RITT*, C. COSSONNET*
}

(Manuscrit reçu le 22 novembre 2000 , accepté le 13 février 2001)

RÉSUMÉ L'évaluation de l'exposition en cas de contamination interne par des composés radiotoxiques, en particulier les actinides, est un des sujets préoccupants en radioprotection de l'homme. Dans le contexte d'une évolution technologique constante dans l'industrie nucléaire et de celle de la réglementation, les moyens permettant d'effectuer en routine la surveillance des travailleurs et d'évaluer l'exposition interne des individus en cas d'accident se doivent d'être performants. La méthode déerite dans ce document est la mesure par ICP-MS (Inductively Coupled Plasma-Mass Spectrometry) de l'uranium dans l'urine après une simple dilution d'un facteur 20 de l'échantillon. L'optimisation des paramètres opératoires a conduit à la proposition d'un protocole analytique permettant la détermination de la concentration massique pour chaque isotope de l'uranium. Les calculs des incertitudes associées et des limites de détection correspondantes sont détaillés.

ABSTRACT Procedure for direct determination of uranium in urine by ICP-MS.

Estimation of the internal exposure of personnel by radioactive substances, especially actinides, is one of the subjects of great importance in human radiological protection. Because of constant changes in industrial processes in the nuclear fuel cycle and new radiological protection regulations, means to perform routine individual monitoring and to estimate internal exposure of workers in an accident situation must be high-efficient. The method described here is the determination of uranium in urine by ICP-MS (Inductively Coupled Plasma-Mass Spectrometry), directly after a simple 20 -fold dilution of the urine samples. Optimisation of the operational parameters has led to propose an analytical procedure that allows the determination of mass concentration for each uranium isotope. Calculations of associated uncertainties and corresponding detection limits are detailed.

\section{Introduction}

Dans le cadre de la surveillance radiotoxicologique des travailleurs du nucléaire exposés à des composés uranifères, il est essentiel de pouvoir mesurer de très faibles niveaux d'activité dans les excrétas. En situation normale, les méthodes utilisées doivent en effet permettre de vérifier le respect des limites de dose imposées par la réglementation et prendre en compte l'évolution des recommandations de la Commission internationale de protection radiologique

* Institut de protection et de sûreté nucléaire, DPHD/SDOS/LEMDI, BP 6, 92265 Fontenay-aux-Roses Cedex, France. 
(CIPR). En présence, ou en cas de suspicion, de contamination interne pouvant induire un risque pour la santé, il est impératif de pouvoir estimer rapidement et de manière fiable son importance.

En pratique courante, les Laboratoires d'analyses et de biologie médicale (LABM) utilisent deux types de méthodes: (i) une mesure globale et (ii) une analyse isotopique. La première méthode, réalisée en général par fluorimétrie classique, donne la concentration massique en uranium total dans l'échantillon. Elle apporte une réponse rapide, permettant une gestion instantanée du risque chimiotoxique en cas d'accident et un tri des agents impliqués. Les volumes prélevés sont suffisamment faibles $(1 \mathrm{ml})$ pour pouvoir démarrer en même temps l'analyse isotopique. Cette seconde méthode permet la détermination de la concentration en activité pour chaque isotope de l'uranium et d'appréhender ainsi le risque radiotoxique. À l'heure actuelle, dans la plupart des laboratoires, cette analyse repose sur des protocoles radiochimiques de purification afin d'extraire l'uranium de la matrice urinaire (1 litre) et de fabriquer une source en couche mince pour une mesure par spectrométrie alpha (Harduin et al., 1996). Sa mise en œuvre est lourde et les délais de réponse plus longs ( 5 jours) que la mesure globale.

Pour répondre à des critères de performances tels que la sensibilité, mais également la rapidité et la simplicité de mise en œuvre, les méthodes utilisant l'ICP-MS peuvent concurrencer celles mettant en jeu la spectrométrie alpha. Depuis le début des années 90, différentes équipes ont étudié l'utilisation de l'ICPMS pour l'analyse de l'uranium dans les urines, en recherchant la préparation de l'échantillon la plus simple possible (Allain et al., 1991 ; Karpas et al., 1996 ; Baglan et al., 1998). Le protocole proposé par Baglan et al. (1999) montre les performances obtenues pour la mesure de l'uranium par ICP-MS après une simple dilution de l'urine.

Afin d'augmenter le nombre et améliorer la qualité des informations que l'on peut obtenir à partir de cette mesure, ce protocole a été optimisé en utilisant l'isotope ${ }^{233} \mathrm{U}$ comme étalon interne et une solution d'uranium enrichi à environ $30 \%$ en ${ }^{235} \mathrm{U}$. Le choix de ces paramètres analytiques permet non seulement de déterminer la concentration massique $\left(\mu \mathrm{g} \mathrm{l}^{-1}\right)$ pour les isotopes 234, 235, 236 et 238 de l'uranium, donc la concentration massique en uranium total, mais également les concentrations en activité correspondantes $\left(\mathrm{mBq}^{-1}\right)$. Le facteur de dilution de l'urine retenu pour ce protocole est égal à 20. Ce document, présenté avec le formalisme d'une méthode d'analyse, décrit la procédure à suivre et explicite les calculs des incertitudes associées aux différentes mesures et les limites de détection correspondantes. 


\section{Méthode}

\subsection{Domaine d'application}

Les limites de détection sont fonction de la sensibilité de l'appareil. Pour une sensibilité de l'ordre de $200000 \mathrm{cps} \mu \mathrm{g} \mathrm{\textrm {I } ^ { - 1 }}$, on obtient :

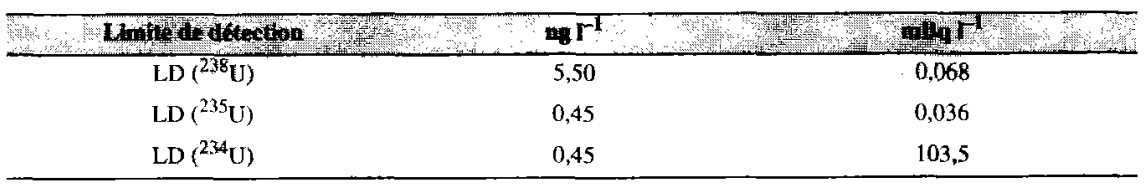

\subsection{Principe}

Les échantillons d'urine sont dilués d'un facteur 20 par $\mathrm{HNO}_{3}$ 0,44 M. L'isotope ${ }^{233} \mathrm{U}$ est ajouté comme étalon interne. La solution est introduite dans I'ICP-MS. Les impulsions correspondant à la masse de l'isotope à doser et à celle de l'étalon interne sont obtenues. Les teneurs en uranium $\left(\mu \mathrm{g} \mathrm{l}^{-1}\right)$ sont calculées à partir des courbes d'étalonnage réalisées avec des solutions étalon.

\subsection{Matériel}

Ont été utilisés un matériel courant de laboratoire et une verrerie jetable pour éviter toute contamination (flacon en HDPE de chez NALGENE), ICP-MS (Plasmaquad $\mathrm{PQ}^{2+}$ VG Elemental, Winsford Cheshire, Angleterre).

\subsection{Réactifs}

Tous les réactifs doivent être garantis purs, de qualité analytique (par exemple NORMATOM ou SUPRAPUR).

R1 : Eau distillée ou purifiée (Milllipore par exemple) de résistivité de l'ordre de $18 \mathrm{M} \Omega \mathrm{cm}$.

R2 : Acide nitrique concentré de pureté Normatom $(\mathrm{d}=1,40$, concentration massique de $65 \%$ ).

R3 : Acide nitrique à $2 \%$ en masse $(0,44 \mathrm{M})$. Solution obtenue par dilution de R2 par un facteur 32,5 .

R4 : Solution à $100 \mathrm{mg} \mathrm{l}^{-1}$ en uranium total (COGEMA Pierrelatte, France). L'uranium doit être enrichi en ${ }^{235} \mathrm{U}$ et l'isotopie certifiée. Prendre un uranium dont l'abondance isotopique en ${ }^{235} \mathrm{U}$ est d'environ $30 \%$, pourcentage 
relativement élevé pour avoir une meilleure statistique de comptage en ${ }^{234} \mathrm{U}$ et ${ }^{235} \mathrm{U}$.

R5 : Solution à $0,1 \mathrm{mg} \mathrm{l}^{-1}$ en uranium total en milieu nitrique préparée par dilution d'un facteur 1000 de R4. La dilution est faite par pesée (voir recommandations particulières $n^{\circ} 1$ ). Utiliser un flacon en HDPE (NALGÈNE), préalablement taré et verser 99,9 $\mathrm{ml}$ de R3. Peser le flacon et verser $0,1 \mathrm{ml}$ de $\mathrm{R} 4$.

R6 : Solution à $10 \mu \mathrm{g} \mathrm{l}^{-1}$ en uranium total en milieu nitrique préparée par dilution d'un facteur 10 de R5. Procéder de la même manière que pour R5 $(90 \mathrm{ml}$ de $\mathrm{R} 3$ et $10 \mathrm{ml}$ de $\mathrm{R} 5$ ).

R7 : Solution d'étalon interne. Préparer une solution de ${ }^{233}$ U (DAMRI, France) en milieu nitrique (R3) à $10 \mu \mathrm{g} \mathrm{I}^{-1}$. La concentration de l'étalon interne n'a pas besoin d'être connue avec une grande précision. Il suffit que toutes les solutions aient la même concentration en étalon interne.

R8 : Urine non contaminée (appelée par la suite le blanc urinaire). On s'affranchit de la présence de traces d'uranium dans cette urine (Sect. 2.6.3., Éq. (4)).

\subsection{Mode opératoire}

\subsubsection{Préparation des solutions}

Les solutions sont préparées par pesée pour minimiser les erreurs (voir recommandations particulières $n^{\circ}$ 1). Les flacons Nalgène sont tarés avant les ajouts et une pesée est réalisée après chaque ajout d'un constituant.

Toutes les solutions (étalon et de mesure) contiennent $0,1 \mu \mathrm{g} \mathrm{l}^{-1}$ de ${ }^{233} \mathrm{U}$ $\left(35,7 \mathrm{~Bq} \mathrm{l}^{-1}\right)$. Les effluents seront donc réservés.

\section{a) Préparation des blancs}

\begin{tabular}{|c|c|c|c|c|c|}
\hline $\begin{aligned} 1 \\
1\end{aligned}$ & 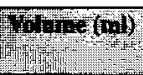 & 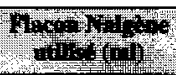 & 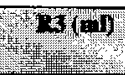 & A & (16) \\
\hline Blanc urinalre & 50 & 60 & 47 & 0,5 & 25 \\
\hline Blanc acide & 50 & 60 & 49,5 & 0,5 & \\
\hline
\end{tabular}

\section{b) Préparation de la gamme d'étalonnage}

Le principe est de préparer des solutions étalon dans un milieu le plus représentatif possible de celui des échantillons. Ces solutions seront donc préparées avec de l'urine non contaminée, diluée 20 fois. 


\begin{tabular}{|c|c|c|c|c|c|c|c|}
\hline Thy & 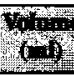 & 1) & 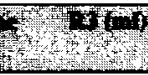 & 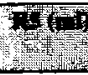 & (3) & 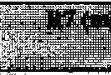 & 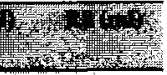 \\
\hline 0,005 & 100 & 125 & 93,95 & & 0,05 & 1 & 5 \\
\hline 0,05 & 50 & 60 & 46,75 & & 0,25 & 0,50 & 2,50 \\
\hline 0,1 & 50 & 60 & 46,50 & & 0,50 & 0,50 & 2,50 \\
\hline 2 & 30 & 30 & 27,60 & 0,60 & & 0,30 & 1,50 \\
\hline
\end{tabular}

Les préparations se faisant par pesée, les valeurs exactes des différentes concentrations sont donc connues.

La composition isotopique de l'uranium utilisé étant connue, une courbe d'étalonnage peut être tracée pour chaque isotope en $\mu \mathrm{gl}^{-1}$.

\section{c) Préparation des échantillons}

Tarer un flacon Nalgène de $60 \mathrm{ml}$ et ajouter $47 \mathrm{ml}$ de $\mathrm{R} 3,0,5 \mathrm{ml}$ de $\mathrm{R} 7$ et $2,5 \mathrm{ml}$ de l'urine à analyser (agiter le flacon avant le prélèvement).

On appellera les solutions obtenues, solutions de mesure, c'est-à-dire les solutions qui sont introduites dans l'ICP-MS, pour éviter toute ambiguité avec les solutions initiales d'urine.

\subsubsection{Mesure}

\section{a) Réglage de l'ICP-MS}

Démarrer le plasma et attendre que le système soit à l'équilibre.

Effectuer les réglages en fonction du mode opératoire propre à l'appareil (puissance du plasma, débit d'argon, débit de prélèvement de l'échantillon) sur une solution multiélémentaire à $1 \mu^{g^{-1}}$ en milieu nitrique.

Vérifier que les performances de l'appareil sont conformes à celles attendues, notamment en terme de sensibilité, de stabilité du signal et du calibrage en masse.

\section{b) Mesure}

Faire toutes les mesures en mode saut de pic « peak jumping » avec trois points par pic et 5 DAC d'écart. Sélectionner les masses 233 (étalon interne) et 234, 235, 236 et 238. Faire 5 acquisitions d'une minute par solution à mesurer.

Ensuite, réaliser la séquence suivante :

- Passer le blanc acide qui détermine la ligne de base (bruit électronique + traces d'uranium dans l'acide nitrique).

- Adapter les réglages au milieu urinaire. Passer la solution étalon à $0,1 \mu \mathrm{g} \mathrm{l}^{-1} \mathrm{en}$ uranium total. Vérifier que la sensibilité reste proche de celle de la solution multiélémentaire utilisée pour les réglages et que le signal est stable. 
- Passer le blanc urinaire et attendre que le signal ne diminue plus. Une fois ce seuil atteint, lancer l'acquisition du blanc urinaire.

- Passer les solutions étalon par ordre croissant jusqu'à celui à $0,1 \mu \mathrm{g} \mathrm{l^{-1 }}$.

- Passer le blanc urinaire.

- Passer les solutions de mesure (échantillons). Entre deux solutions de mesure, intercaler le blanc urinaire (pour palier au phénomène d'effet mémoire).

- Passer la solution étalon à $2 \mu \mathrm{g} \mathrm{l}^{-1}$.

\subsection{Expression des résultats}

Les acquisitions terminées, il faut procéder aux calculs suivants :

- calcul des facteurs de dilution pour toutes les solutions préparées ;

- correction des variations de sensibilité de l'appareil à l'aide de l'étalon interne ;

- détermination des droites d'étalonnage ;

- calcul de la concentration en uranium dans les solutions de mesure puis, dans l'urine ;

- détermination des incertitudes ;

- détermination des limites de détection.

\subsubsection{Facteurs de dilution}

À l'aide des pesées, il faut déterminer les facteurs de dilution $(D)$ de chaque constituant.

La détermination de $D$ permet :

- pour l'étalon interne, de vérifier que sa concentration est identique dans tous les flacons. Le cas échéant, on peut procéder à une correction si les écarts observés sont trop importants (voir Sect. 2.6.2.) ;

- pour les solutions étalon, de connaître la concentration exacte en uranium total et donc d'avoir une droite d'étalonnage très précise ;

- pour les solutions de mesure, de remonter à la concentration initiale dans l'urine.

Soit $D_{i}(x)$, le facteur de dilution du constituant « $i \gg$ d'une solution $« x \gg$, contenant $n$ constituants. On peut écrire :

$$
D_{i}(x)=\frac{\sum_{j=1}^{n} \frac{m_{j}}{\rho_{j}}}{\frac{m_{i}}{\rho_{i}}}
$$

avec $\rho_{i}$ : densité du constituant $i$ et $m_{i}$ : masse du constituant $i$ (pesée). 


\subsubsection{Correction par rapport à l'étalon interne $\left({ }^{233} U\right)$}

Il faut tout d'abord déterminer les facteurs de dilution pour l'étalon interne $\left({ }^{233} \mathrm{U}\right)$ dans toutes les solutions ( $y$-compris les solutions étalon). On peut alors corriger les nombres de coups par seconde (cps) obtenus pour chaque autre isotope de l'uranium dans toutes les solutions, pour s'affranchir de la variation de sensibilité de l'appareil au cours du temps. Cette correction permet également d'annuler les effets de matrice d'un échantillon à l'autre.

Pour cela, on prend une solution de référence, en général le blanc urinaire (le premier flacon mesuré), et on corrige tous les résultats obtenus pour les autres solutions par rapport à cette référence, avec la formule suivante :

$$
N_{x}^{u}(\mathrm{cor})=\frac{N_{x}^{u} N_{\mathrm{ref}}^{233}}{N_{x}^{233}}
$$

avec

$N_{x}^{u} \quad$ : nombre de coups mesuré pour l'isotope $u$ dans la solution de mesure $x$;

$N_{x}^{u}($ cor $)$ : nombre de coups corrigé pour l'isotope $u$ dans la solution de mesure $x$;

$N_{\text {rel }}^{233} \quad$ : nombre de coups mesuré pour l'isotope 233 dans la solution choisie comme référence ;

$N_{x}^{233} \quad$ : nombre de coups mesuré pour l'isotope 233 dans la solution de mesure $x$.

Remarque : Si la concentration en ${ }^{233} \mathrm{U}$ n'est pas la même dans toutes les solutions (i.e. $D$ différent de 20 ), il faut alors tenir compte du facteur de dilution vrai pour la correction. L'équation (2) devient :

$$
N_{x}^{u}(\text { cor })=\frac{N_{x}^{u} N_{\text {ref }}^{233} D_{233}(\text { ref })}{N_{x}^{233} D_{233}(x)}
$$

avec $D_{233}$ (ref) : facteur de dilution du constituant ${ }^{233} \mathrm{U}$ dans la solution choisie comme référence ;

$D_{233}(x)$ : facteur de dilution du constituant ${ }^{233} \mathrm{U}$ dans la solution $x$. 


\subsubsection{Détermination des droites d'étalonnage}

La première étape pour la détermination des droites d'étalonnage est de connaître aussi précisément que possible la concentration de chaque isotope dans les solutions étalon. Ceci est possible grâce à la connaissance des trois paramètres suivants :

- la concentration en uranium total dans la solution R4 (matériau de référence) ;

- l'isotopie de la solution R4;

- les facteurs de dilution de l'uranium dans les solutions étalon.

Le détail des calculs pour la détermination de la masse de chaque isotope d'uranium présent dans le matériau de référence et dans les solutions étalon est donné en remarques particulières $\mathrm{n}^{\circ} 2$.

Ces données permettent de déterminer une droite d'étalonnage par isotope. La concentration dans les solutions à mesurer sera donc déterminée de manière indépendante par isotope.

Les courbes [nombre de coups nets par seconde $N^{u}$ en cps] en fonction de la concentration [ $C^{u}$ en $\mu \mathrm{g} \mathrm{I}^{-1}$ ] sont tracées, pour chaque isotope, avec $N^{u}$ égal à :

$$
N_{\text {étalon }}^{u}(\text { nets })=N_{\text {étalon }}^{u}(\text { cor }) \dot{-} N_{\text {blanc_urinaire }}^{u}(\text { cor }) .
$$

En appliquant la méthode des moindres carrés (régression linéaire), les équations des droites d'étalonnage sont de la forme :

$$
N_{\text {étalon }}^{u}(\text { nets })=C_{\text {étalon }}^{u} \cdot m^{u}
$$

avec

$C_{\text {étalon }}^{u}$ : concentration de l'isotope $u$ en $\mu \mathrm{g} \mathrm{I}^{-1}$ dans la solution étalon;

$m^{u} \quad$ : coefficient de la droite d'étalonnage. C'est également la sensibilité de l'appareil pour l'isotope $u$ en cps/ $\mu \mathrm{g}^{-1}$.

Le matériau de référence utilisé pour les solutions étalon ne contenant pas de ${ }^{236} \mathrm{U}$, la droite d'étalonnage pour cet isotope ne peut être tracée. Nous prenons la moyenne des coefficients obtenus pour ${ }^{235} \mathrm{U}$ et ${ }^{238} \mathrm{U}$. 
À titre indicatif, sont données ci-dessous des valeurs de sensibilité déterminées pour le matériau de référence :

\begin{tabular}{|c|c|c|}
\hline Why & 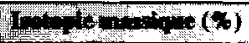 & 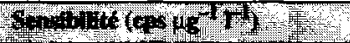 \\
\hline${ }^{238} \mathrm{U}$ & 67,0513 & 206362 \\
\hline${ }^{235} \mathrm{U}$ & 32,5119 & 225029 \\
\hline${ }^{234} \mathrm{U}$ & 0,4368 & 231957 \\
\hline
\end{tabular}

\subsubsection{Concentration en uranium (uranium pondéral)}

Les droites d'étalonnage permettent de déterminer la concentration massique de chaque isotope dans les solutions de mesure. En sommant ces concentrations, on obtient la quantité totale d'uranium présent dans l'urine.

Pour chaque isotope des solutions de mesure, au nombre de coups mesuré, puis corrigé, est soustrait le nombre de coups du blanc acide, pour éliminer la contribution du bruit électronique et des traces d'uranium dans l'acide nitrique. Le nombre de coups nets pour l'isotope $u$ dans la solution de mesure $x, N_{x}^{u}$ (nets), est donc égal à :

$$
N_{x}^{u}(\text { nets })=N_{x}^{u}(\text { cor })-N_{\text {blanc_acide }}^{u}(\text { cor })
$$

À l'aide des droites d'étalonnage, on a :

$$
C_{x}^{u}=\frac{N_{x}^{u}(\text { nets })}{m^{u}}
$$

et en tenant compte des facteurs de dilution, la concentration dans l'urine $x$, pour l'isotope $u$, est égale à :

$$
C_{\text {urine_x }}^{u}=D_{\text {urine }}(x) C_{x}^{u}
$$

La concentration en uranium total en $\mu \mathrm{g} \mathrm{l}^{-1}$ dans l'urine $x$ est :

$$
C_{\text {urine } \_x}=\sum_{u} C_{\text {urine } \_x}^{u} .
$$

En résumé, la démarche suivie conduit à l'expression suivante :

$$
C_{\text {urine_t }_{-}}=D_{\text {urine }}(x) N_{\text {ref }}^{233} \sum_{u}\left(\frac{N_{x}^{u}}{N_{x}^{233}}-\frac{N_{\text {blanc_acide }}^{u}}{N_{\text {blanc_acide }}^{233}}\right) \frac{l}{m^{u}} .
$$


Cette équation n'est valable que si la concentration en ${ }^{233} \mathrm{U}$ est la même dans toutes les solutions, sinon il faut reprendre le calcul avec l'équation (3).

\subsubsection{Incertitudes}

En appliquant la propagation des erreurs pour des variables indépendantes à l'équation (8), on obtient l'équation (11).

Pour des simplifications d'écriture, on note $A$ le facteur suivant :

$$
A=\frac{N_{b}^{u}}{N_{b}^{233}} \frac{N_{x}^{233}}{N_{x}^{u}}
$$

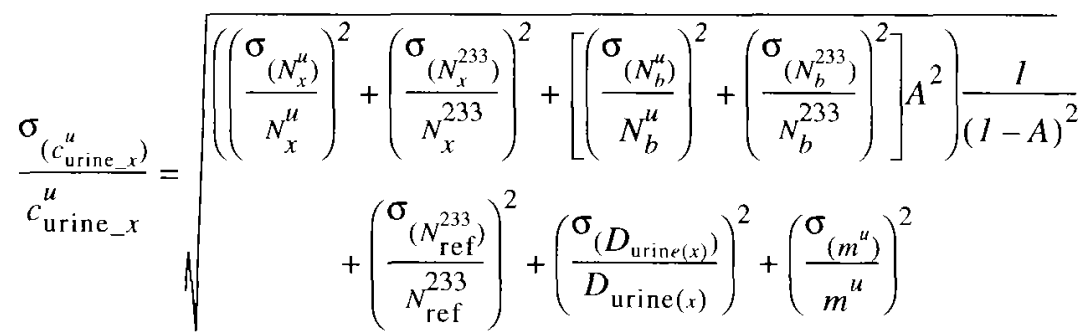

$\sigma(x)$ est l'incertitude absolue de la grandeur $x$ et $b$ est le blanc acide.

Dans l'équation (11), il y a 3 types d'incertitude :

- les incertitudes sur les mesures;

- les incertitudes sur les facteurs de dilution, donc sur les pesées ;

- les incertitudes sur les coefficients des droites d'étalonnage.

\section{a) Incertitude de mesure}

L'incertitude sur la mesure, donc sur les grandeurs $N$, est égale à l'écart-type sur les 5 acquisitions.

\section{b) Incertitude sur les facteurs de dilution}

En appliquant la propagation des erreurs à l'équation (1) et en faisant l'hypothèse que les densités des réactifs sont bien connues et leur incertitude négligeable, l'incertitude sur $D_{i}(x)$ est donnée par l'équation (12). 
L'équation (1) donnait :

$$
\begin{aligned}
D_{i}(x)=\frac{\sum_{j=1}^{n} \frac{m_{j}}{\rho_{j}}}{\frac{m_{i}}{\rho_{i}}} \text { ou } D_{i}(x)=1+\frac{\sum_{j=1, j \neq i}^{n} \frac{m_{j}}{\rho_{j}}}{\frac{m_{i}}{\rho_{i}}} \\
\sigma\left(D_{i}\right)=\frac{\rho_{i}}{m_{i}} \sqrt{\frac{\left(\sum_{j=1, j \neq i} \frac{m_{j}}{\rho_{j}}\right)^{2}}{\left(\frac{m_{i}}{\rho_{i}}\right)^{2}} \frac{\sigma^{2} m_{i}}{\rho_{i}^{2}}+\sum_{j=1, j \neq i}^{n} \frac{\sigma^{2} m_{j}}{\rho_{j}^{2}} .}
\end{aligned}
$$

L'incertitude sur les pesées est égale à $5 \times 10^{-4} \mathrm{~g}$. On peut donc simplifier l'équation (12):

$$
\sigma\left(D_{i}\right)=5 \times 10^{-4} \frac{\rho_{i}}{m_{i}} \sqrt{\frac{\left.\sum_{j=1, j \neq i} \frac{m_{j}}{\rho_{j}}\right)^{2}}{m_{i}^{2}}+\sum_{j=1, j \neq i} \frac{1}{\rho_{j}^{2}}} .
$$

Cette incertitude est calculée en considérant les densités comme des constantes. Or, la densité de l'urine peut être variable. En évaluant cette variabilité à $0,5 \%$ et en simplifiant les équations précédentes, on peut estimer l'incertitude globale (notée dans ce cas, $\sigma^{\prime}$ ) sur les facteurs de dilution pour l'urine, de la manière suivante :

$$
\frac{\sigma\left(D_{\text {urine }}\right)}{D_{\text {urine }}} \quad \Longrightarrow \quad \frac{\sigma^{\prime}\left(D_{\text {urine }}\right)}{D_{\text {urine }}}=\sqrt{\left(\frac{\sigma\left(D_{\text {urine }}\right)}{D_{\text {urine }}}\right)^{2}+\left(\frac{0,5}{100}\right)^{2}} .
$$

\section{c) Incertitude sur les coefficients des droites d'étalonnage}

Excel donne l'écart-type sur le coefficient de la droite. Cependant, il est plus juste d'ajouter l'incertitude sur la concentration de l'isotope dans la solution étalon correspondante, ce qui donne :

$$
\frac{\sigma\left(m^{u}\right)}{m^{u}} \quad \Longrightarrow \quad \frac{\sigma^{\prime}\left(m^{u}\right)}{m^{u}}=\sqrt{\left(\frac{\sigma\left(m^{u}\right)}{m^{u}}\right)^{2}+\left(\frac{\sigma\left(C_{\text {êtalon }}^{u}\right)}{C_{\text {étalon }}^{u}}\right)^{2}} .
$$

Remarque : Dans l'expression de l'incertitude globale (Éq. (11)), il y a un terme en dénominateur avec des rapports de nombres de coups (facteur $A$ ). Quand le nombre de 
coups d'un échantillon est très faible, voisin du blanc, on constate que ce terme tend vers 0 , donc que l'incertitude sur le résultat devient très élevée.

Pour terminer, l'incertitude sur la concentration en uranium total est :

$$
\sigma\left(C_{\text {urine }_{-} x}\right)=\sqrt{\sum_{u} \sigma^{2}\left(C_{\text {urine } \_}^{u}\right)} .
$$

\subsubsection{Limites de détection}

Comme les droites d'étalonnage ont été tracées par isotope, il est possible de calculer la limite de détection par isotope. La limite de détection est la plus faible concentration qu'il est possible de déterminer. Or la concentration pour un isotope donné est liée au nombre de coups par la relation :

$$
C^{u}=\frac{N^{u}-N_{\text {blanc_urinaire }}^{u} .}{m^{u}}
$$

Retirer le blanc urinaire est un choix plus restrictif que de retirer le blanc acide.

Le nombre de coups minimum, significativement différent des fluctuations statistiques du blanc, pour un isotope donné, est la limite de détection, $N_{L D}^{u}$ :

$$
N_{L D}^{u}=N_{\text {blanc_urinaire }}^{u}+k \sigma_{\text {blanc_urinaire }}^{u}
$$

avec $\sigma_{\text {blanc_urinaire }}^{u}$ : écart-type sur les 5 mesures de l'isotope $u$ du blanc urinaire et $k$ : facteur de sécurité, pris, en général, égal à 3 en ICP-MS.

La limite de détection en concentration pour la solution de mesure, d'après l'équation (17), est égale à :

$$
L D^{u}=3 \frac{\sigma_{\text {blanc_urinaire }}^{u}}{m^{u}}
$$

Soit, dans l'urine (dilution 20) en $\mu \mathrm{g}^{-1}$, pour l'isotope $u$ :

$$
L D_{\text {urine }}^{u}=20 L D^{u}
$$

Avec la démarche proposée dans ce mode opératoire, tous les résultats exprimés ci-dessus en $\mu \mathrm{g} \mathrm{I}^{-1}$, pour les isotopes $234,235,236$ et 238 de l'uranium, peuvent être traduits en $\mathrm{mBq}^{-1}$. 


\subsection{Remarques particulières}

\section{No 1. Pesée}

Pour les dilutions effectuées par pesée, il faut suivre certaines conditions opératoires afin que la pesée soit précise :

- la balance utilisée n'a pas besoin d'être étalonnée car on n'effectue que des pesées relatives. Par contre pour éviter les variations de pression atmosphérique, il est souhaitable que la préparation se déroule dans la même journée ;

- on doit peser une masse suffisamment grande par rapport à l'incertitude de mesure, qui est de $5 \times 10^{-4} \mathrm{~g}$. La mesure d'une masse inférieure à $0,02 \mathrm{~g}$ (correspondant à environ $20 \mu \mathrm{l}$ ) entraînerait une incertitude relative trop importante $(2,5 \%)$;

- tous les réactifs doivent être à température ambiante ;

- il est nécessaire de connaître la densité des réactifs :

$\mathrm{HNO}_{3} 2 \%: d=1,010$;

Urine : la densité peut varier d'un individu à l'autre, mais en moyenne elle est de 1,021.

La détermination du facteur de dilution se fait par le rapport des volumes des différents réactifs.

\section{$\mathbf{N}^{\circ}$ 2. Calcul de l'isotopie massique}

La composition isotopique permet de connaître, par l'intermédiaire du calcul de la masse molaire, l'isotopie massique.

Cas du matériau de référence utilisé ST 024 de la COGEMA. Le certificat de garantie donne l'isotopie atomique.

\begin{tabular}{|c|c|c|c|c|}
\hline 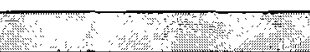 & SWU & ${ }^{23} \mathrm{U}$ & 250 & ${ }^{28} \mathrm{U}$ \\
\hline lsotopie atomique $(\%)$ & $0,4424 \pm 0,0088$ & $32,7890 \pm 0,0328$ & $\leq 0,0003$ & $66,7686 \pm 0,0340$ \\
\hline Masse molaire $\mathrm{MO}^{*}$ (uma) & & 237,047123 & & \\
\hline Isotopie massique ${ }^{* *}(\%)$ & $0,4368 \pm 0,0088$ & $32,5119 \pm 0,0328$ & - & $67,0513 \pm 0,0340$ \\
\hline
\end{tabular}

* Á partir de l'isotopie atomique et de la masse atomique de chaque ísotope $u$, on calcule la masse molaire, $M O$, de cet uranium : $M O=\sum_{u} m_{u} a_{u}$, avec $m_{u}$ : masse atomique en umi de l'isotope $u ; a_{u}$ abondance atomique de l'isotope $u$.

** L'abondance massique, $A_{u}$, de l'isotope $u$ est alors égale à : $A_{u}=\frac{m_{u} a_{u}}{M O}$.

Le matériau a été dissous dans $100 \mathrm{ml}$ de $\mathrm{HNO}_{3} 2 \mathrm{M}$ Normatom, puis la solution R4 a été préparée. Les facteurs de dilution permettent de connaître la 
concentration massique de chaque isotope d'uranium dans chacune des solutions étalon.

\section{Commentaires et conclusions}

Pour la mise en œuvre du protocole, l'urine est diluée d'un facteur 20. La charge saline de l'urine affectant la stabilité de l'appareil, une dilution d'un facteur 100 peut, en fonction de la situation rencontrée (routine, tri, accident), être un bon compromis entre la sensibilité et la stabilité. Le même protocole peut être suivi, il suffit de modifier les volumes des différents réactifs.

Dans la majorité des travaux antérieurs, les auteurs utilisent ${ }^{209} \mathrm{Bi}$ comme étalon interne. Le choix d'ajouter l'isotope ${ }^{233} \mathrm{U}$, isotope de l'élément à déterminer et absent des différents composés industriels d'uranium, permet une meilleure correction de la variation de la sensibilité en fonction du milieu. De plus, quand une purification chimique est nécessaire avant la mesure par ICP-MS, ${ }^{233} \mathrm{U}$ peut être utilisé comme traceur pour déterminer le rendement chimique.

Mais le principal avantage du protocole proposé est la détermination de la concentration par isotope. Dans les précédents protocoles, la droite d'étalonnage était tracée uniquement pour ${ }^{238} \mathrm{U}$ à l'aide d'une solution d'uranium appauvri ou naturel. Pour calculer la concentration massique totale, on faisait l'hypothèse que les sensibilités étaient égales pour tous les isotopes à celle en ${ }^{238} \mathrm{U}$. Pour les isotopes mineurs, cette approximation pouvait entraîner une incertitude élevée. Avec ce nouveau protocole, les droites d'étalonnage obtenues pour chaque isotope permettent le calcul de la concentration massique par isotope. Il suffit ensuite de sommer sur tous les isotopes mesurés pour obtenir la concentration massique totale en uranium. Le protocole permet d'obtenir des résultats significatifs ou les limites de détection en $\mu \mathrm{g} \mathrm{l}^{-1}$ par isotope et donc, de transformer toutes les valeurs en $\mathrm{mBq}^{-1}$.

D’une manière générale, deux cas de figures sont rencontrés :

- $1^{\text {er }}$ cas : seuls les nombres d'impulsions pour les isotopes 235 et 238 sont significatifs. Si la valeur du rapport 235/238 permet de conclure que l'on est en présence d'uranium appauvri ou naturel, on a tous les renseignements; concentrations par isotope et en uranium total, en $\mu \mathrm{g}$ et en $\mathrm{mBq}$. Si la valeur du rapport 235/238 permet de conclure que l'on est en présence d'uranium enrichi, on peut calculer la concentration massique en uranium total. Par contre, en fonction du niveau rencontré, il faudra réaliser une purification chimique, pour gagner un facteur de concentration, pour déterminer la concentration en activité pour l'isotope 234, voire pour l'isotope 236. 
- $2^{\mathrm{e}}$ cas : les nombres d'impulsions pour tous les isotopes sont significatifs. Connaissant les sensibilités de l'appareil pour chaque isotope, on a tous les renseignements, en concentration massique et en activité, par isotope et donc, en uranium total.

En conclusion, les performances en termes de sensibilité et de rapidité de l'ICPMS ont été prouvées. Comme la plupart des protocoles déjà mis au point avec cette technique, le protocole proposé dans ce document apporte un nombre de renseignements beaucoup plus important que la mesure globale classique par fluorimétrie et évite la longueur de l'analyse isotopique mettant en jeu la spectrométrie alpha. Il nécessite un minimum de préparation de l'échantillon et permet un délai de réponse court (quelques heures). L'ajout de ${ }^{233} \mathrm{U}$ comme étalon interne et l'utilisation d'une solution d'uranium enrichi à $30 \%$ en ${ }^{235} \mathrm{U}$ pour réaliser des droites d'étalonnage par isotope $(234,235,238$ et par approximation 236) sont les originalités de ce protocole. Le fait de déterminer la sensibilité de l'appareil pour chaque isotope est une démarche plus rigoureuse et permet l'obtention de résultats plus justes. Les résultats obtenus par ce protocole de mesure directe de l'uranium dans l'urine par ICP-MS dans ces conditions opératoires regroupent en fait les résultats obtenus classiquement en mettant en cuvre deux techniques (par exemple fluorimétrie + spectrométrie alpha). Cette méthode analytique qui est qualifiée de rapide et globale, qualités que l'on associe généralement à une mesure «grossière»pour des applications de tri, peut également permettre une mesure «fine» (analyse isotopique), en fonction des composés et des niveaux de concentrations rencontrés.

\section{RÉFÉRENCES}

Allain P., Berre S., Prenel-Cabic A., Mauras Y., Delaporte T., Cournot A. (1991) Investigation of the direct determination of uranium in plasma and urine by inductively coupled plasma mass spectrometry, Anal. Chim. Acta 251, 183-185.

Baglan N., Bérard P., Trompier F., Cossonnet C., Le Guen B. (1998) How to reduce the uncertainties of commited dose derived from urinary measurements investigation of new protocols using ICP-MS, Radiat. Protect. Dosim. 79, 477-480.

Baglan N., Cossonnet C., Trompier F., Ritt J., Bérard P. (1999) Implementation of ICP-MS protocols for uranium urinary measurements in worker monitoring, Health Phys. 77 (4), 455-461.

Harduin J.C., Peleau B., Levavasscur D. (1996) Analytical determination of actinides in biological samples, Radioprotection 31 (2), 229-245.

Karpas Z., Halicz L., Roiz J., Marko R., Katorza E., Lorber A., Goldbar Z. (1996) Inductively coupled plasma mass spectrometry as a simple, rapid, and inexpensive method for determination of uranium in urine and fresh water: a comparison with LIF, Health Phys. 71 (6), 879-885. 